\section{Gastrectomía totalmente laparoscópica por neoplasias gástricas. Experiencia en un centro público}

\author{
ENRIQUE NORERO ${ }^{1}$, SERGIO BÁEZ ${ }^{1}$, EDUARDO BRICEÑO ${ }^{1}$, \\ CRISTIAN MARTÍNEZ ${ }^{1}$, MARCO CERONI ${ }^{1}$, ALEX ESCALONA ${ }^{1}$, \\ GLORIA AGUAYO ${ }^{2}$, PAULINA GONZÁLEZ $Z^{1, a}$, FERNANDO ARAOS ${ }^{3}$, \\ ALFONSO CALVO $^{1}$, ALFONSO DÍAZ ${ }^{1}$, EDUARDO VIÑUELA ${ }^{1}$
}

\section{Totally laparoscopic gastrectomy for the treatment of gastric tumors}

Background: The laparoscopic approach for the treatment of gastric tumors has many advantages. Aim: To evaluate the results of a laparoscopic gastrectomy program developed in a public hospital. Patients and Methods: Retrospective review of epidemiological, perioperative and follow-up data of patients who were treated with a laparoscopic gastrectomy due to gastric tumors between 2006 and 2013. A totally laparoscopic technique was used for all cases. Complications were evaluated according to the Clavien-Dindo classification. Results: Fifty one patients, aged 65 (36-85) years, underwent a laparoscopic gastrectomy. In 22 patients a total gastrectomy was performed. Conversion rate to open surgery was $8 \%$. Operative time was 330 (90-500) min and bleeding was 200 (20-500) $\mathrm{ml}$. Median hospital stay was 7 (3-37) days. Postoperative morbidity was present in $17(33 \%)$ patients, 3 (6\%) patients had complications grade 3 or higher and one patient died (1.9\%). Tumor pathology was adenocarcinoma in 39 patients. A complete resection was achieved in 97\%. Twenty nine patients (74\%) with gastric adenocarcinoma had early gastric cancer and $84 \%$ of patients were in stage one. Median lymph node count was 24. Median follow-up was 26 (1-91) months. There was no cancer related mortality among patients subjected to a curative resection. Overall survival for patients with adenocarcinoma was 92\% at 3 years. Conclusions: This study supports the feasibility and safety of a laparoscopic gastrectomy program in a public hospital; with low morbidity, adequate lymph node dissection and long-term survival. This approach must be considered an option for selected patients with gastric cancer.

(Rev Med Chile 2015; 143: 281-288)

Key words: Gastrectomy; Minimally invasive surgical procedures; Stomach neoplasms.

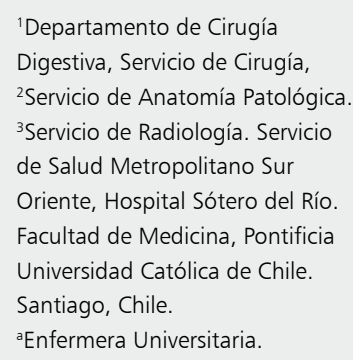

Conflictos de intereses: ninguno que declarar.

Recibido el 19 de enero de 2014, aceptado el 26 de enero de 2015 .

Correspondencia a:

Dr. Enrique Norero Muñoz.

Concha y Toro 3459, Puente Alto,

Santiago de Chile.

Teléfono: 56-02-3536601.

enorero@uc.cl

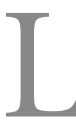

a cirugía laparoscópica del cáncer gástrico ha presentado un desarrollo exponencial en la últimas dos décadas. Este abordaje ofrece significativas ventajas al disminuir el trauma operatorio: menor sangrado intraoperatorio, menor dolor, menos íleo postoperatorio, menores complicaciones, especialmente de la herida y respiratorias, logrando una estadía acortada en el hospital ${ }^{1-6}$. En el largo plazo existe una menor posibilidad de hernias incisionales y obstrucciones intestinales derivadas de adherencias y los resultados oncológicos, para pacientes seleccionados, son comparables al de pacientes operados por vía abierta $^{5,6}$. 
Sin embargo, esta técnica tiene algunas desventajas: una mayor necesidad de insumos y tecnología en el pabellón, y requiere un mayor entrenamiento de los cirujanos debido a la mayor dificultad técnica, particularmente asociada a la linfadenectomía y la reconstrucción del tubo digestivo. Además, requiere una cuidadosa selección de los pacientes, lo que potencialmente limita su uso masivo.

La mayor experiencia en este abordaje viene de Japón y Corea, con varias decenas de miles de pacientes operados ${ }^{7}$. En nuestro país la experiencia es limitada ${ }^{8,9}$.

El objetivo de este trabajo es evaluar los resultados de un programa de gastrectomía laparoscópica para el tratamiento de tumores gástricos desarrollado en un hospital público chileno. Los objetivos específicos fueron evaluar las complicaciones de la técnica y la sobrevida alejada.

\section{Pacientes y Métodos}

Estudio retrospectivo a partir de una base de datos prospectiva. Se incluyó a todos los pacientes sometidos a una gastrectomía por tumores gástricos por vía laparoscópica en forma consecutiva, desde julio de 2006 hasta diciembre de 2013. Las cirugías fueron realizadas por los mismos cirujanos que realizan la cirugía abierta por tumores gástricos.

La evaluación preoperatoria incluyó una endoscopia digestiva alta y biopsia, exámenes de laboratorio general, electrocardiograma y evaluación nutricional. El estudio de diseminación se realizó con una ecografía abdominal, radiografía de tórax y una tomografía computada (TAC) de tórax, abdomen y pelvis. Los casos se discutieron en reunión semanal clínico-radiológica. No se utilizó tratamiento adyuvante preoperatorio.

En la selección de los pacientes para esta técnica, evitamos pacientes ASA 3 descompensados, particularmente con patología cardiaca, respiratoria y renal grave. Incluimos pacientes con laparotomías supraumbilicales. Se seleccionaron preferentemente pacientes con índice masa corporal (IMC) hasta 35 o hasta 30 en los casos de obesidad abdominal. Se incluyeron pacientes con adenocarcinoma y otras histologías. En la primera mitad de la serie, desde la primera gastrectomía laparoscópica hasta la número 25 , se incluyeron en forma exclusiva pacientes con cáncer gástrico incipiente (compromiso de mucosa o submuco- sa), no candidatos a tratamiento endoscópico, y en la segunda mitad, desde la cirugía número 26, ampliamos la indicación hasta tumores con compromiso de la muscular propia y subserosa, de diámetro máximo de $4 \mathrm{~cm}$, sin compromiso de la serosa ni ganglionar de acuerdo a la TAC. Todos los casos con sospecha de invasión de órganos vecinos, infiltración esofágica o duodenal fueron excluidos, y se trataron con cirugía abierta.

\section{Técnica quirúrgica}

El paciente se ubicó en decúbito supino. Neumoperitoneo con $\mathrm{CO}_{2}$ a $15 \mathrm{mmHg}$. Utilizamos entre 5 a 7 trocares y óptica de $30^{\circ}$. Se resecó el epiplón, excepto por algunos casos de cáncer incipiente. Se seccionó el duodeno con sutura mecánica. La linfadenectomía se ha modificado desde disección D1 a D2 en la medida que se han incluido casos con cáncer gástrico avanzado ${ }^{10}$. Se extrajo la pieza por una incisión transversa suprapúbica de $4 \mathrm{~cm}$. Se utilizó una técnica totalmente laparoscópica para la reconstrucción. La gastrectomía subtotal se realizó con una Y de Roux o Billroth 2, con una anastomosis gastroyeyunal mecánica. La reconstrucción en gastrectomía total se realizó con una Y de Roux y una anastomosis esofagoyeyunal (AEY) manual intracorpórea en todos los pacientes, excepto en 2 casos en que se utilizó un sistema de sutura mecánica circular introducido por la boca. Al final de la cirugía se instalaron 2 drenajes en gastrectomía total y uno en gastrectomía subtotal. En los casos de tumores con histología distinta a adenocarcinoma se realizó una linfadenectomía limitada y se favorecieron las resecciones locales por laparoscopia.

El postoperatorio del paciente se realizó con una observación en la recuperación, trasladándose a la sala general a las 12-24 h. Se inició la alimentación al tercer día postoperatorio. En caso de gastrectomía total, previo a la realimentación, se realizó un estudio contrastado para descartar filtración de la AEY. El paciente fue dado de alta sin drenajes una vez que fue capaz de tolerar un régimen papilla.

Para el análisis de las complicaciones se utilizó la clasificación de Clavien-Dindo. Las complicaciones tipo 3 o superior se consideraron graves ${ }^{11}$. Se consideró la morbilidad y mortalidad postoperatoria hasta 30 días de la cirugía o durante la hospitalización. Se consideró morbilidad respiratoria cuando el paciente presentó disnea, 
tos o fiebre y se realizó un estudio de imágenes que demostró atelectasia, neumonía o derrame pleural. Se utilizó el TNM 7a edición ${ }^{12}$. Los casos con cáncer gástrico avanzado o ganglios positivos se discutieron en Comité Oncológico para decidir tratamiento adyuvante.

Se siguieron a todos los pacientes con controles regulares en el policlínico, con exámenes de imágenes y endoscopia. Se actualizó el seguimiento a través del Registro Civil hasta diciembre de 2013, con un seguimiento de $100 \%$.

Análisis estadístico: Se utilizó el programa Minitab 15 y SPSS 20. Los datos se expresaron en medianas (rango) o promedios \pm desviación estándar. Para comparación de datos se utilizaron test de $\chi^{2}$ y t de Student. Se realizó una curva de sobrevida global para los casos de adenocarcinoma gástrico con Kaplan-Meier, incluyendo la mortalidad operatoria en la evaluación de sobrevida alejada.

\section{Resultados}

De un total de 561 gastrectomías realizadas durante el período considerado, se utilizó el abordaje laparoscópico en 51 casos (9\%). Se incluyeron 49 pacientes, en 2 pacientes se realizó una segunda gastrectomía laparoscópica a los 3 meses debido a un margen microscópico positivo. La mediana de edad fue 65 años (36-85). El 94\% de los pacientes se clasificaron como ASA 1 o 2 . Once pacientes (22\%) presentaban una colecistectomía abierta previa. La ubicación del tumor fue similar en los 3 tercios del estómago y se operaron 2 casos de cáncer incipiente de unión gastroesofágica (Tabla 1).

Se realizaron 22 gastrectomías totales y 20 gastrectomías subtotales distales (Tabla 1). En 4 casos $(8 \%)$ se decidió una conversión a cirugía abierta, por causas que se detallan en la Tabla 1. Se realizó una linfadenectomía D2 en 17 casos y menor a D2 en los casos restantes.

El tiempo operatorio fue de $330 \mathrm{~min}$ (90-500). Para los pacientes sometidos a una gastrectomía total este fue de $385 \mathrm{~min}$ (180-500), gastrectomía subtotal distal $330 \mathrm{~min}$ (210-420), gastrectomía subtotal en cuña 130 min (90-210). El sangrado fue de $200 \mathrm{ml}$ (20-500).

La mediana de estadía hospitalaria fue de 7 días (3-37). Para los pacientes sometidos a gastrectomía subtotal en cuña esta fue de 5 días (3-7), en gastrectomía subtotal distal de 6 días (4-29) y gastrectomía total 9 días (6-37).
Se registró morbilidad postoperatoria en 17 casos (33\%) (Tabla 2), 3 de las cuales fueron graves $(6 \%)$ (Clavien 3 o mayor) (Tabla 3). La única mortalidad postoperatoria estuvo dada por un paciente con fistula del muñón duodenal y de la AEY, que fue reoperado, evolucionó con falla orgánica múltiple y falleció el día 11 postoperatorio. En otros 2 pacientes se requirió una reintervención; en un paciente se realizó un drenaje laparoscópico de un absceso relacionado a una fístula duodenal el día 12 postoperatorio; el segundo caso requirió tratamiento endoscópico con inyectoterapia debido a sangrado digestivo posterior a gastrectomía subtotal al segundo día postoperatorio. Se produjeron 2 fistulas de la AEY (8\%) y 3 fístulas del muñón duodenal (7\%) (Tabla 2). La AEY en estos 2 pacientes con filtración fue realizada con técnica manual intracorpórea en el primer caso y con el sistema de sutura mecánica circular

\section{Tabla 1. Datos demográficos y perioperatorios de pacientes sometidos a gastrectomía laparoscópica}

\begin{tabular}{|ll|}
\hline $\mathrm{n}$ & 51 \\
\hline Hombre/Mujer & $20(39 \%) / 31(61 \%)$ \\
\hline Edad (años) & $65(36-85)$ \\
IMC $\left(\mathrm{kg} / \mathrm{mt}^{2}\right)$ & $27(19-39)$ \\
\hline ASA & \\
1 & $17(33 \%)$ \\
2 & $31(61 \%)$ \\
3 & $3(6 \%)$ \\
\hline Laparotomías previas & $26(51 \%)$ \\
Laparotomías supraumbilicales & $11(22 \%)$ \\
Ubicación del tumor gástrico & \\
Tercio inferior & $15(29 \%)$ \\
Tercio medio & $16(31 \%)$ \\
Tercio superior & $18(35 \%)$ \\
$\quad$ Unión gastroesofágica & $2(4 \%)$ \\
Cirugías realizadas & \\
Gastrectomía total & $22(43 \%)$ \\
Gastrectomía subtotal distal & $20(39 \%)$ \\
Gastrectomía subtotal cuña & $7(14 \%)$ \\
Totalización & $2(4 \%)$ \\
Conversión a cirugía abierta & $4(8 \%)$ \\
Adherencias y obesidad central & $1(2 \%)$ \\
Malrotación intestinal & $1(2 \%)$ \\
Falla en la sección duodenal & $1(2 \%)$ \\
Invasión tumoral del duodeno & $1(2 \%)$ \\
\hline
\end{tabular}


Tabla 2. Complicaciones postoperatorias precoces de pacientes sometidos a gastrectomía laparoscópica

\begin{tabular}{|lcl|}
\hline Complicación & n & (\%) \\
\hline Fistula anastomosis esofagoyeyunal & 2 & $(8)^{*}$ \\
Fistula duodenal & 3 & $(7)^{* *}$ \\
\hline Fistula pancreática & 1 & $(2)$ \\
Hematoma herida & 2 & $(4)$ \\
Sangrado digestivo & 2 & $(4)$ \\
Retención gástrica & 6 & $(22)^{* * *}$ \\
Colección abdominal & 2 & $(4)$ \\
ITU & 1 & $(2)$ \\
Colitis por Clostridium & 3 & $(6)$ \\
Trombosis subclavia & 1 & $(2)$ \\
TVP & 1 & $(2)$ \\
\hline Total pacientes con complicaciones & 17 & $(33)$ \\
\hline
\end{tabular}

*Fístula esofagoyeyunal 2/24 esofagoyeyuno anastomosis realizadas. ${ }^{* *}$ Fistula duodenal 3/42 muñones duodenales. ***Retención gástrica 6/27 gastrectomías subtotales realizadas. ITU: Infección del tracto urinario. TVP: Trombosis venosa profunda. introducido por la boca en el segundo caso. Entre las complicaciones tardías destaca sólo un caso de obstrucción intestinal por adherencias en un paciente con laparotomías previas y un caso de hernia incisional. No encontramos diferencias en el porcentaje de complicaciones entre la primera y segunda mitad de esta serie ( $33 \%$ vs $33 \%, \mathrm{p}=\mathrm{NS}$ ).

La evaluación de anatomía patológica confirmó 39 casos de adenocarcinoma, 7 tumores GIST, 1 tumor fusocelular, 1 schwanoma y 1 lipoma gigante. Se logró una resección R0 en 92\% de los pacientes con diagnóstico de adenocarcinoma. En 2 pacientes con cáncer gástrico incipiente, tratados con gastrectomías subtotales distales, el borde proximal fue focalmente positivo, por lo que se sometieron a una totalización de la gastrectomía por vía laparoscópica a los 3 meses de la primera cirugía, en ninguno de ellos se encontró tumor en el remanente gástrico resecado. Con esto el R0 final aumentó a 97\%. En un paciente con cáncer avanzado se realizó una cirugía R2, de carácter paliativo, debido al hallazgo intraoperatorio de diseminación peritoneal limitada (Tabla 4). Los tumores GIST presentaron una mediana de tama-

Tabla 3. Gravedad de las complicaciones precoces según Clavien-Dindo

\begin{tabular}{|c|c|c|}
\hline Gravedad & n (\%) & Tratamiento de la complicación \\
\hline $\begin{array}{l}\text { Clavien } 1 \\
\text { Fístula pancreática } \\
\text { Hemorragia digestiva } \\
\text { Hematoma herida } \\
\text { Retención gástrica }\end{array}$ & $\begin{array}{l}9(18) \\
1 \\
1 \\
2 \\
6\end{array}$ & $\begin{array}{l}\text { Manejo con drenaje } \\
\text { Suspender heparina } \\
\text { Manejo local herida } \\
\text { Mantención de SNG }\end{array}$ \\
\hline $\begin{array}{l}\text { Clavien } 2 \\
\text { ITU } \\
\text { Colitis por Clostridium } \\
\text { Colección abdominal } \\
\text { Fístula AEY } \\
\text { Fístula Duodenal } \\
\text { Trombosis subclavia } \\
\text { TVP }\end{array}$ & $\begin{array}{c}10(20) \\
1 \\
3 \\
2 \\
1 \\
1 \\
1 \\
1\end{array}$ & $\begin{array}{l}\text { Antibióticos } \\
\text { Antibióticos } \\
\text { Antibióticos } \\
\text { NPTC, manejo con drenaje } \\
\text { Antibióticos, manejo con drenaje } \\
\text { Anticoagulación, antibióticos } \\
\text { Anticoagulación }\end{array}$ \\
\hline $\begin{array}{l}\text { Clavien 3A } \\
\text { Hemorragia digestiva }\end{array}$ & $\begin{array}{ll}1 & (2) \\
1 & \end{array}$ & Tratamiento endoscópico \\
\hline $\begin{array}{l}\text { Clavien 3B } \\
\quad \text { Fístula duodenal }\end{array}$ & $\begin{array}{ll}1 & (2) \\
1 & \end{array}$ & Reoperación, aseo y drenaje \\
\hline $\begin{array}{l}\text { Clavien } 5 \\
\text { Fístula AEY y Duodeno }\end{array}$ & $\begin{array}{ll}1 & (2) \\
1 & \end{array}$ & Reoperación, UCI, Fallece \\
\hline
\end{tabular}

NPTC: Nutrición parenteral total central. SNG: sonda nasogástrica. AEY: Anastomosis esofagoyeyunal. ITU: Infección del tracto urinario. TVP: Trombosis venosa profunda. 
ño de $2,8 \mathrm{~cm}(2-4)$ y todos presentaban entre $0-3$ mitosis en 10 campos de aumento mayor. Para las histologías distintas a adenocarcinoma la cirugía fue R0 en todos los casos. Los casos de cirugía R1 y R2 se produjeron en la primera mitad de la serie.

La evaluación postoperatoria del TNM para adenocarcinoma informó 29 cánceres incipientes (T1) (74\%) y 10 avanzados (T2-T3-T4a) (26\%). En 5 pacientes (13\%) existió compromiso ganglionar. El recuento ganglionar global para los casos de adenocarcinoma fue 24 ganglios (4-72). Este fue mayor en gastrectomía total 29 (7-61) y menor en gastrectomía subtotal 22 (4-72). En cáncer avanzado se obtuvo un recuento de 37 ganglios (10-61) y en cáncer incipiente de 20 (4-73). La distribución por etapas fue predominante para etapa $1(84 \%)$ (Tabla 4). Se decidió quimioterapia-radioterapia postoperatoria en 2 pacientes con etapa 2B. Uno de los pacientes rechazó el tratamiento adyuvante y la segunda paciente lo completó sin inconvenientes.

La mediana de seguimiento es de 26 meses (1-91). Hasta la fecha han fallecido 5 pacientes, uno de ellos en el postoperatorio inmediato. La paciente sometida a gastrectomía paliativa falleció a los 3 meses. Los 3 pacientes restantes fallecieron a los 6, 62 y 78 meses de la cirugía por causas no relacionadas a su tumor: infarto agudo al miocardio y 2 neumonías. Del total de pacientes sólo registramos un caso de recidiva locoregional, a los 22 meses de una gastrectomía total por adenocarcinoma avanzado; esta paciente sigue viva y realizando tratamiento con quimioterapia. $\mathrm{La}$
Tabla 4. Evaluación de anatomía patológica de los pacientes con adenocarcinoma

\begin{tabular}{|lrr|}
\hline & n & (\%) \\
T T1a (mucosa) & & \\
T1b (submucosa) & 20 & $(51 \%)$ \\
T2 (muscular) & 9 & $(23 \%)$ \\
T3 (subserosa) & 5 & $(13 \%)$ \\
T4a (serosa) & 1 & $(3 \%)$ \\
N & 4 & $(10 \%)$ \\
N0 & & \\
N1 & 34 & $(87 \%)$ \\
N2 & 3 & $(7 \%)$ \\
N3 & 1 & $(3 \%)$ \\
Etapa & 1 & $(3 \%)$ \\
1a & & \\
1b & & $(71 \%)$ \\
2b & 28 & $(13 \%)$ \\
3a & 5 & $(10 \%)$ \\
4 & 4 & $(3 \%)$ \\
Status de la cirugía & 1 & $(3 \%)$ \\
R0 & 1 & \\
R1* & & $(92 \%)$ \\
R2** & 36 & $(3 \%)$ \\
\hline
\end{tabular}

*Gastrectomías subtotales que se totalizaron con un resultado final de cirugía R0. ${ }^{* * *}$ Gastrectomía paliativa por diseminación peritoneal.

sobrevida global en pacientes con adenocarcinoma fue de $92 \%$ a 36 meses (Figura 1). Ningún paciente con histología no-adenocarcinoma ha presentado mortalidad ni recidiva hasta la fecha.

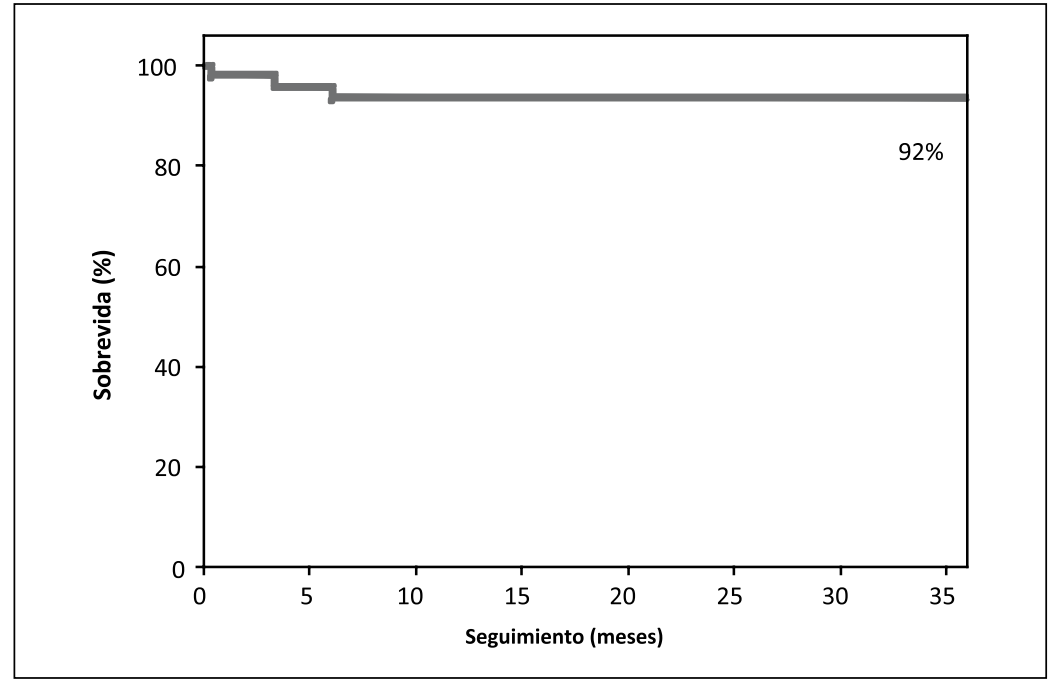

Figura 1. Sobrevida global de pacientes sometidos a gastrectomía laparoscópica por adenocarcinoma gástrico. 


\section{Discusión}

Este trabajo muestra la serie clínica más grande y con mayor seguimiento de gastrectomía totalmente laparoscópica por tumores gástricos, realizada en nuestro país en el sistema público. Destacan un bajo porcentaje de complicaciones graves, una corta estadía hospitalaria y una excelente sobrevida alejada.

La morbilidad de una gastrectomía, descrita en series nacionales e internacionales, es de $19-43 \%{ }^{14-19}$. La morbilidad global de esta serie es comparable a esos datos; sin embargo, las complicaciones graves que requirieron una reintervención se produjeron en sólo 6\%. La mortalidad también fue baja, comparada a series de gastrectomía abierta que describen entre 1-11\% de mortalidad ${ }^{14-19}$. Recientemente reportamos nuestra experiencia de los últimos 16 años de gastrectomía abierta (Norero E. et al. Observaciones no publicadas, 2013), en la cual tuvimos 5,4\% de mortalidad postoperatoria, una morbilidad de $29 \%$ y una estadía postoperatoria de 11 días. A pesar de que los grupos no son comparables y los pacientes con abordaje laparoscópico son seleccionados, vemos una disminución de la mortalidad a 1,9\% y una disminución de la estadía a 7 días con el abordaje laparoscópico. En trabajos aleatorizados y controlados, existe evidencia de una significativa menor morbilidad global y respiratoria con cirugía laparoscópica ${ }^{2,20}$; nuestra serie lo apoya porque ningún paciente presentó complicaciones respiratorias, probablemente debido a menor dolor y una mejor recuperación respiratoria ${ }^{1}$. Los meta análisis realizados también apoyan una significativa menor tasa de complicaciones con este abordaje mínimamente invasivo ${ }^{6,21,22}$.

Entre los otros beneficios de la laparoscopia están un menor sangrado intraoperatorio, menor respuesta inflamatoria sistémica ${ }^{1}$, mejor calidad de vida a corto plazo $^{23}$ y menor estadía hospitalaria ${ }^{1-6,20,24}$. En esta serie la estadía hospitalaria de 7 días se compara favorablemente con series de cirugía abierta, favorecido por menos íleo postoperatorio y una realimentación $\mathrm{precoz}^{8}$.

Una de las desventajas de este abordaje es el mayor tiempo operatorio. La duración de 5,5 h de cirugía en nuestra serie es más largo que una cirugía abierta. Según la literatura el tiempo operatorio aumenta aproximadamente una hora y este efecto se demuestra consistentemente entre las distintas publicaciones ${ }^{6,22,24}$.
La linfadenectomía se considera uno de los pilares fundamentales de la cirugía por cáncer gástrico. Este es uno de los puntos críticos, ya que requiere trabajar en cercanía a grandes vasos (arteria hepática, tronco celiaco y arteria esplénica) sin producir lesiones de éstos y recolectando todo el tejido linfático que los rodea. En esta serie iniciamos nuestra experiencia con cáncer incipiente, situación en la cual una linfadenectomía D1 es adecuada ${ }^{10}$, y fuimos avanzando hacia una linfadenectomía D2, la cual se realizó en 17 casos, sin morbilidad asociada a esta etapa de la cirugía. La literatura apoya que se puede realizar una linfadenectomía equivalente a la cirugía abierta, favorecida por la magnificación y la excelente visión laparoscópica ${ }^{6,25}$. Nuestros datos apoyan una linfadenectomía adecuada, con una mediana de 24 ganglios por cirugía.

En gastrectomías subtotales distales, la complejidad de la reconstrucción es baja. De acuerdo con esto no tuvimos complicaciones de la anastomosis gastroyeyunal. En gastrectomía total la situación es distinta ya que se debe realizar una anastomosis con el esófago, trabajando en la parte alta del abdomen. Para esta última anastomosis se han descrito múltiples técnicas, sin que exista un consenso sobre la mejor alternativa ${ }^{13,21,26-29}$. En esta serie tuvimos 2 fistulas de la AEY. Si bien son sólo 2 casos con fistula, esto es $8 \%$, que se encuentra en el límite alto aceptado para esta cirugía, por lo que creemos que se deben realizar trabajos comparativos para definir la mejor técnica de anastomosis, con baja filtración y estenosis, y reproducible, de manera de no perder los beneficios de la técnica laparoscópica.

Los resultados oncológicos son adecuados, logrando una cirugía R0 final en 97\% de los pacientes y un adecuado recuento ganglionar. Esto determina una excelente sobrevida a 3 años. La literatura apoya la seguridad oncológica de esta cirugía, especialmente para cáncer gástrico incipiente ${ }^{1-3,6,30-32}$, que son la mayoría de nuestros casos.

Para cáncer gástrico avanzado, nuestro grupo de pacientes es pequeño para generar conclusiones. Sin embargo, los resultados son alentadores con sólo un caso de recidiva. En la literatura existe un trabajo aleatorizado controlado que muestra una sobrevida equivalente a la cirugía abierta ${ }^{5}$ varios meta análisis recientes, que apoyan una equivalencia oncológica, manteniendo los beneficios perioperatorios ${ }^{20,24,33,34}$. 


\section{Limitaciones del estudio}

Esta experiencia se logró en un grupo seleccionado de pacientes, sin un grupo control adecuado de cirugías abiertas para comparación, y es un análisis retrospectivo. Esta es la experiencia de un centro y no necesariamente aplicable a otros hospitales, con equipamiento y entrenamiento diferentes ${ }^{35}$.

Nuestro estudio muestra que es factible y seguro desarrollar un programa de esta complejidad en un hospital público. Su implementación debe tomar en cuenta una adecuada selección de los pacientes, instrumental y equipamiento adecuado, entrenamiento y una curva de aprendizaje progresiva. Pensamos que su implementación es necesaria, debido a la alta incidencia de cáncer gástrico en nuestro país, la evidencia actual y los resultados preliminares descritos en esta serie, considerando que la mayoría de los pacientes son beneficiarios del sistema público.

Agradecimientos: Agradecemos el interés y la ayuda de nuestras instrumentistas quirúrgicas (Gladys Arenas y Silvana Trapp), paramédicos del Servicio de Endoscopia (María Alicia Correa y Claudia Velásquez), la Nutricionista Lucía Marinkovich y el apoyo de la Dirección del Hospital al desarrollo de este proyecto.

\section{Referencias}

1. Kitano S, Shiraishi N, Fujii K, Yasuda K, Inomata M, Adachi Y. A randomized controlled trial comparing open vs laparoscopy-assisted distal gastrectomy for the treatment of early gastric cancer: an interim report. Surgery 2002; 131 (1 Suppl): S306-11.

2. Lee JH, Han HS, Lee JH. A prospective randomized study comparing open vs laparoscopy-assisted distal gastrectomy in early gastric cancer: early results. Surg Endosc 2005; 19 (2): 168-73.

3. Hayashi H, Ochiai T, Shimada H, Gunji Y. Prospective randomized study of open vs laparoscopy-assisted distal gastrectomy with extraperigastric lymph node dissection for early gastric cancer. Surg Endosc 2005; 19 (9): 11726.

4. Kim HH, Hyung WJ, Cho GS, Kim MC, Han SU, Kim $\mathrm{W}$, et al. Morbidity and mortality of laparoscopic gastrectomy versus open gastrectomy for gastric cancer: an interim report-a phase III multicenter, prospective, randomized trial (KLASS Trial). Ann Surg 2010; 251 (3): 417-20.
5. Huscher CG, Mingoli A, Sgarzini G, Sansonetti A, Di Paola M, Recher A, et al. Laparoscopic versus open subtotal gastrectomy for distal gastric cancer: five-year results of a randomized prospective trial. Ann Surg 2005; 241 (2): 232-7.

6. Zeng YK, Yang ZL, Peng JS, Lin HS, Cai L. Laparoscopyassisted versus open distal gastrectomy for early gastric cancer: evidence from randomized and nonrandomized clinical trials. Ann Surg 2012; 256 (1): 39-52.

7. Kitano S, Shiraishi N, Uyama I, Sugihara K, Tanigawa N. Japanese laparoscopic surgery study group. A multicenter study on oncologic outcome of laparoscopic gastrectomy for early cancer in japan. Ann Surg 2007; 245 (1): 68-72.

8. Moisan F, Norero E, Slako M, Varas J, Palominos G, Crovari F, et al. Completely laparoscopic versus open gastrectomy for early and advanced gastric cancer: a matched cohort study. Surg Endosc 2012; 26 (3): 661-72.

9. Escalona A, Báez S, Pimentel F, Calvo A, Boza C, Viñuela E, et al. Laparoscopic gastrectomy for gastric cancer. Rev Chil Cir 2008; 60 (3): 188-93.

10. Japanese Gastric Cancer Association. Japanese gastric cancer treatment guidelines 2010 (ver. 3). Gastric Cancer 2011; 14 (2):113-23.

11. Dindo D, Demartines N, Clavien PA. Classification of surgical complications: a new proposal with evaluation in a cohort of 6336 patients and results of a survey. Ann Surg 2004; 240 (2): 205-13.

12. Edge S, Byrd DR, Compton CC, Fritz AG, Greene FL, Trotti A. AJCC Cancer Staging Manual. 7th ed. New York, NY: Springer-Verlag; 2010.

13. La Femina J, Viñuela EF, Shattner MA, Gerdes H, Strong VE. Esophagojejunal reconstruction after total gastrectomy for gastric cancer using a transorally inserted anvil delivery system. Ann Surg Oncol 2013; 20 (9): 2975-83.

14. Llanos O, Guzmán S, Pimentel F, Ibáñez L, Duarte I. Results of surgical treatment of gastric cancer. Dig Surg 1999; 16 (5): 385-8.

15. García CC, Benavides CC, Apablaza SP, Rubilar PO, Covacevich SR, Peñaloza PM, et al. Surgical treatment of gastric cancer: results in 423 cases. Rev Med Chile 2007; 135 (6): 687-95.

16. Csendes A, Burdiles P, Rojas J, Braghetto I, Díaz JC, Maluenda F. A prospective randomized study comparing D2 total gastrectomy versus D2 total gastrectomy plus splenectomy in 187 patients with gastric carcinoma. Surgery 2002; 131 (4): 401-7.

17. Oñate-Ocaña LF, Cortés-Cárdenas SA, Aiello-Crocifoglio V, Mondragón-Sánchez R, Ruiz-Molina JM. Preoperative multivariate prediction of morbidity after 
gastrectomy for adenocarcinoma. Ann Surg Oncol 2000; 7 (4): 281-8.

18. Bonenkamp JJ, Songun I, Hermans J, Sasako M, Welvaart K, Plukker JT, et al. Randomized comparison of morbidity after D1 and D2 dissection for gastric cancer in 996 Dutch patients. Lancet 1995; 345 (8952): 745-8.

19. Cuschieri A, Fayers P, Fielding J, Craven J, Bancewicz J, Joypaul, et al. Postoperative morbidity and mortality after D1 and D2 resections for gastric cancer: preliminary results of the MRC randomized controlled surgical trial. The Surgical Cooperative Group. Lancet 1996; 347 (9007): 995-9.

20. Zhao Y, Yu P, Hao Y, Qian F, Tang B, Shi Y, et al. Comparison of outcomes for laparoscopically assisted and open radical distal gastrectomy with lymphadenectomy for advanced gastric cancer. Surg Endosc 2011; 25 (9): 2960-6.

21. Haverkamp L, Weijs T, van der Sluis PC, van der Tweel I, Ruurda J, van Hillegersberg R. Laparoscopic total gastrectomy versus open total gastrectomy for cancer: a systematic review and meta-analysis. Surg Endosc 2013; 27 (5): 1509-20.

22. Viñuela EF, Gonen M, Brennan MF, Coit DG, Strong VE. Laparoscopic versus open distal gastrectomy for gastric cancer: a meta-analysis of randomized controlled trials and high-quality nonrandomized studies. Ann Surg 2012; 255 (3): 446-56.

23. Kim YW, Baik YH, Yun YH, Nam BH, Kim DH, Choi IJ, et al. Improved quality of life outcomes after laparoscopy-assisted distal gastrectomy for early gastric cancer: results of a prospective randomized clinical trial. Ann Surg 2008; 248 (5): 721-7.

24. Hwang SI, Kim HO, Yoo CH, Shin JH, Son BH. Laparoscopic-assisted distal gastrectomy versus open distal gastrectomy for advanced gastric cancer. Surg Endosc 2009; 23 (6): 1252-8.

25. Hyung WJ, Lim JS, Song J, Choi SH, Noh SH. Laparoscopic spleen-preserving splenic hilar lymph node dissection during total gastrectomy for gastric cancer. J Am Coll Surg 2008; 207 (2): e6-e11.

26. Usui S, Nagai K, Hiranuma S, Takiguchi N, Matsumoto
A, Sanada K. Laparcopy-assisted esophoenteral anastomosis using endoscopic purse-string suture instrument "endo-psi (II)" and circular stapler. Gastric Cancer 2008; 11 (4): 233-7.

27. Kinoshita T, Oshiro T, Ito K, Shibasaki H, Okazumi S, Katoh R. Intracorporeal circular-stapled esophagojejunostomy using hand-sewn purse string suture after laparoscopic total gastrectomy. Surg Endosc 2010; 24 (11): 2908-12.

28. Okabe H, Obama K, Tsunoda S, Tanaka E, Sakai Y. Advantage of completely laparoscopic gastrectomy with linear stapler reconstruction. A long-term follow-up study. Ann Surg 2014; 259 (1): 109-16.

29. Facy O, Arru L, Azagra J. Intestinal anastomosis after laparoscopic total gastrectomy. J Visceral Surg 2012; 149 (3): e179-e84.

30. Mochiki E, Kamiyama Y, Aihara R, Nakabayashi T, Asao T, Kumano H. Laparoscopic assisted distal gastrectomy for early gastric cancer: five years' experience. Surgery 2005; 137 (3): 317-22.

31. Lee SE, Kim YW, Lee JH, Ryu KW, Cho SJ, Lee JY, et al. Developing an institutional protocol guideline for laparoscopy-assisted distal gastrectomy. Ann Surg Oncol 2009; 16 (8): 2231-6.

32. An JY, Heo GU, Cheong JH, Hyung WJ, Choi SH, Noh SH. Assessment of open versus laparoscopy-assisted gastrectomy in lymph node-positive early gastric cancer: a retrospective cohort analysis. J Surg Oncol 2010; 102 (1): 77-81.

33. Oiu J, Pankaj P, Jiang H, Zeng Y, Wu H. Laparoscopy versus open distal gastrectomy for advanced gastric cancer: a systematic review and meta-analysis. Surg Laparosc Endosc Percutan Tech 2013; 23 (1): 1-7.

34. Chen K, Xu XM, Mou YP, Pan Y, Zhou YC, Zhang RC, et al. Systematic review and meta-analysis of laparoscopic and open gastrectomy for advanced gastric cancer. World J Surg Oncol 2013; 11: 182.

35. Kim MC, Jung GJ, Kim HH. Learning curve of laparoscopy-assisted distal gastrectomy with systemic lymphadenectomy for early gastric cancer. World J Gastroenterol 2005; 11 (47): 7508-11. 